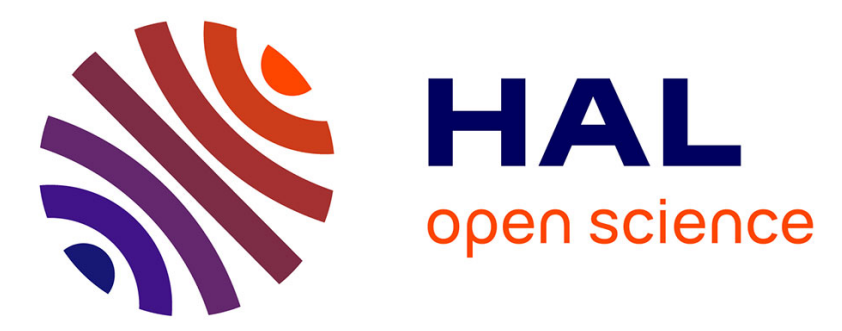

\title{
Electromagnetic Flow Control: Characteristic Numbers and Flow Regimes of a Wall-Normal Actuator
}

\author{
Jean-Paul Thibault, Lionel Rossi
}

\section{To cite this version:}

Jean-Paul Thibault, Lionel Rossi. Electromagnetic Flow Control: Characteristic Numbers and Flow Regimes of a Wall-Normal Actuator. Journal of Physics D: Applied Physics, 2003, 36, pp.2559-2568. 10.1088/0022-3727/36/20/021 . hal-00260443

\section{HAL Id: hal-00260443 \\ https://hal.science/hal-00260443}

Submitted on 7 Feb 2020

HAL is a multi-disciplinary open access archive for the deposit and dissemination of scientific research documents, whether they are published or not. The documents may come from teaching and research institutions in France or abroad, or from public or private research centers.
L'archive ouverte pluridisciplinaire HAL, est destinée au dépôt et à la diffusion de documents scientifiques de niveau recherche, publiés ou non, émanant des établissements d'enseignement et de recherche français ou étrangers, des laboratoires publics ou privés. 


\title{
Electromagnetic flow control: characteristic numbers and flow regimes of a wall-normal actuator
}

\author{
Jean-Paul Thibault and Lionel Rossi \\ LEGI $^{1}$, BP 53, 38041 Grenoble, France \\ E-mail: jean-paul.thibault@inpg.fr and lionel.rossi@netcourrier.com
}

\begin{abstract}
Electromagnetic (EM) flow control of boundary layer refers to the use of 'wall-flush' electrodes ( $\boldsymbol{j}$, current density) and 'sub-surface' magnets $(\boldsymbol{B}$, magnetic induction) used in combination to create local Lorentz body forces $(\boldsymbol{j} \times \boldsymbol{B})$. In the present application the working fluid is seawater. Close to the boundary wall, these $\boldsymbol{j} \times \boldsymbol{B}$ forces can act directly on velocity and vorticity. In this paper, the characterization of a wall-normal EM actuator (i.e. $\boldsymbol{j} \times \boldsymbol{B}$ forces are mainly wall-normal above the central axis of the actuator) is considered. An idealized inertial and integral approach leads to the definition of characteristic EM numbers in term of velocity, time, acceleration and length-scales. These numbers are useful in introducing an EM parameter similar to the Froude number. Furthermore, two asymptotic EM flow regimes, which depend on flow velocity and on EM forces intensity, are also discussed.
\end{abstract}

\section{Introduction}

The work presented in this paper was developed within the context of electromagnetic (EM) flow control in seawater where Lorentz forces are imposed near the wall by means of EM actuators. It is known from the literature that EM flow control can reduce turbulent intensity and drag [1] as well as prevent flow separation $[2,3]$.

The EM actuator is a novel concept that permits the direct application of local three-dimensional Lorentz forces within the flow. These local EM body forces are associated with additional forcing terms $(\boldsymbol{j} \times \boldsymbol{B})$ in Navier-Stokes equations. Typically, an EM actuator comprises a pair of wall-flush electrodes and a pair of sub-surface magnets. The configuration of electrodes and magnets on the wall surface is such that the curl of $\boldsymbol{j} \times \boldsymbol{B}$ is non-zero in the vicinity of the actuator $[4,5]$. This means that the EM forces acting on the fluid near the wall can pump or deflect the flow as well as impose vorticity sources. Velocity and/or vorticity fields are therefore modified by EM control either

1 LEGI is a joint laboratory of the Centre National de la Recherche Scientifique (CNRS), Université Joseph Fourier (UJF) and the Institut National Polytechnique de Grenoble (INPG). directly during activation or indirectly due to the persistence of induced velocity (wall-normal component and wall jets) and vorticity [6].

One of the key questions regarding this concept of flow control is the determination of length-scales and timescales appropriate to the EM forcing and their comparison to the mean flow scales. In the following the description of an EM actuator and the basic equations governing EM flow control in seawater are given. Following this, a number of characteristic parameters, derived from an idealized model are computed, and an EM Froude similarity is suggested and discussed. Finally, the EM Froude similarity is validated with experimental data using various electrical duty cycles and two different EM actuators.

\section{Description of the EM actuator and basic equations}

The combination 'wall-flush' electrodes ( $\boldsymbol{j}$, current density) and 'sub-surface' magnets (B, magnetic induction) allows to create local Lorentz body forces $(\boldsymbol{j} \times \boldsymbol{B})$ within seawater boundary layers (see figure $1(a)$ ). Close to the wall, these 
$\boldsymbol{j} \times \boldsymbol{B}$ forces are able to act directly on velocity and vorticity components. Grosso modo, above the centre of the EM actuator, the magnetic field lines and electric field lines intersect each other perpendicularly and are parallel to the wall. Therefore, the Lorentz forces generated by the interaction of these two fields are mainly normal to the wall (as in figure $1(b)$ ). In most of the volume above the actuator, $\operatorname{curl}(\boldsymbol{j} \times \boldsymbol{B})$ is non-zero, which results in the imposition of vorticity sources within the flow. These sources are distributed all around the edges of the EM actuator (figure 1(c)) [6]. By using permanent magnets the intensity and sign of $\boldsymbol{j} \times \boldsymbol{B}$ forces is directly controlled by the electrical power supplied to the electrodes.

To begin the analysis, the appropriate equations are given in table 1. Seawater is an electrolyte, but it is idealized here as a medium with a poor bulk conductivity $\sigma$. The governing equations for the fluid are (1) continuity and (2) the Navier-Stokes equations including the extra EM term due to the Lorentz forces. The vorticity equation (3) is nothing more than the curl of (2). The existence of the right-hand side term: $\operatorname{curl}(\boldsymbol{j} \times \boldsymbol{B})$ demonstrates that EM forces can act as a vorticity source. Equation (4) for the magnetic induction, $\boldsymbol{B}$, reduces to the Laplace equation in the steady state when $\mu \sigma$ is very small. This corresponds to the use of permanent magnets and the very poor conductivity of seawater which gives a very low value to the magnetic Reynolds number (the ratio of magnetic convection to magnetic diffusion). The constitutive equation for the current density $j$ is given by Ohm's law (5) where $\boldsymbol{u} \times \boldsymbol{B}$ is the electromotive field and $\boldsymbol{E}$ the electric field imposed at the electrodes. In the present case, the current density must be relatively high in order to produce strong EM forces. In fact, due to the moderate induction offered by the permanent magnets, the imposed electric field $\boldsymbol{E}$ has to be much larger than the induced electric field $\boldsymbol{u} \times \boldsymbol{B}$ leading to the indicated simplifications. Finally, equations (6) express the conservation of magnetic induction and of electric current.

Both $\boldsymbol{j}$ and $\boldsymbol{B}$ are independent of the flow and consequently the EM force $\boldsymbol{j} \times \boldsymbol{B}$ also has the same property. The EM force distribution therefore depends only on the actuator geometry. In addition, $\boldsymbol{B}$ is produced by the permanent magnets and the EM force intensity is fixed by the electric power supply (applied current and time of activation).

The remarkable fact that the EM force distribution is independent of the flow, places EM flow control out of the conventional problem of magnetohydrodynamics (where $\boldsymbol{J} \times \boldsymbol{B}$ forces are directly dependent on the flow). In addition, this independence offers the possibility to design EM actuators aiming specific goals. For example, the size of an EM actuator might also be fitted to the size of a typical structure present in turbulent boundary layers (micro-actuator) (see Robinson [7], Adrian et al [8]), as to a larger scale like the spacing between packets of structures (macro-actuator) (see Meng [9], Zoo [10], Rossi [4]). (a)

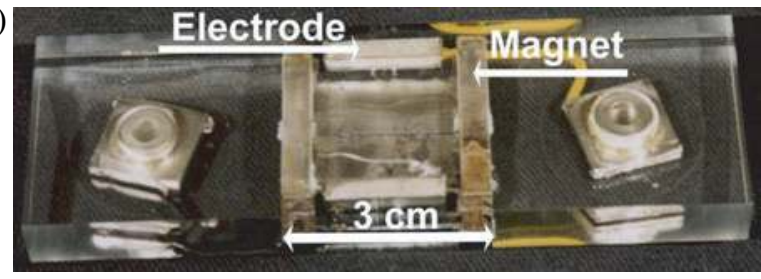

(c)

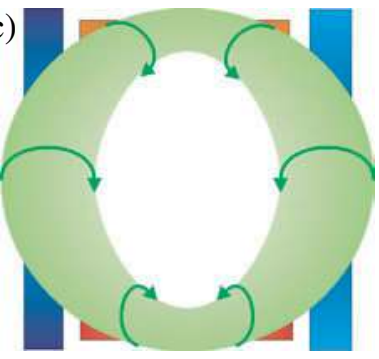

(b)

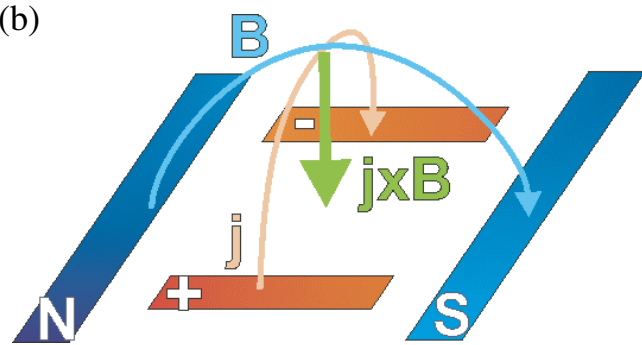

Figure 1. Wall-normal actuator: (a) photograph of the 1999 EM actuator, $(b)$ schematic of magnet and electrode arrangement and associated EM forces, $(c)$ sources of EM vorticy in the boundary layer due to Lorentz forces.

Table 1. Fluid, magnetic and electric equations used in seawater EM flow control.

\begin{tabular}{ll}
\hline \multicolumn{1}{c}{ Fluid equations } & \multicolumn{1}{c}{ Magnetic induction equation and Ohm's law } \\
\hline $\operatorname{div} \boldsymbol{u}=0$ & (1) $\frac{\partial \boldsymbol{B}}{\partial t}=\operatorname{curl}(\boldsymbol{u} \times \boldsymbol{B})+\frac{1}{\mu \sigma} \nabla^{2} \boldsymbol{B} \Rightarrow \nabla^{2} \boldsymbol{B} \approx 0$ \\
$\rho \frac{\mathrm{d} \boldsymbol{u}}{\mathrm{d} t}+\nabla P+\rho \boldsymbol{g}=\mu \nabla^{2} \boldsymbol{u}+\boldsymbol{j} \times \boldsymbol{B}$ & (2) $\boldsymbol{j}=\sigma(\boldsymbol{E}+\boldsymbol{u} \times \boldsymbol{B}) \rightarrow \boldsymbol{j} \approx \sigma \boldsymbol{E}$ \\
$\rho \frac{\mathrm{d} \omega}{\mathrm{d} t}=\rho(\omega \cdot \nabla) \boldsymbol{u}+\mu \nabla^{2} \omega+\operatorname{curl}(\boldsymbol{j} \times \boldsymbol{B})$ & (3) $\quad \operatorname{div} \boldsymbol{B}=0$ and $\operatorname{div} \boldsymbol{j}=0$ \\
\hline
\end{tabular}




\section{Description of the EM forces and characteristic scales}

\subsection{A description of the EM force for an actuator acting normal to the wall}

Due to the special influence of the magnets and electrodes, the EM force field has a rather complicated three-dimensional shape. To a good approximation, the force distribution can be computed using the analytical solution given by Akoun and Yonnet [11]. Basically, it assumes a uniform distribution of electric charge on the electrodes and a uniform distribution of magnetic charge density on the magnets. The model also supposes a uniform electrochemical potential at the surface of the electrodes [13]. The electrochemical reaction results in a difference between the potential of the electrode and the potential of the flow very close to the electrode (over a distance corresponding to the diffusion layer). This over-potential depends on the electrode material and the local concentration of the reacting species and current density. Thus, in the present case a constant over-potential is assumed.

The EM forces computed via this analytical solution are three-dimensional and decrease rapidly with distance from the maximum value at the wall [4]. More precisely, the computed three-dimensional EM force distribution (see figure 2(a)) shows that the forces are wall-normal above the centre of actuator and are three-dimensionally centripetal all around. At the centre of the actuator, the intensity of the EM forces decreases rapidly with distance from the wall. Quantitative values across the actuator are shown on figure $2(b)$ for different $y$ values.

\subsection{Characteristic scales}

The characteristic length-scales of an EM actuator are clearly $L_{\mathrm{E}}$ (electrode spacing) and $L_{\mathrm{M}}$ (magnet spacing) on the wall (figure 3). However, unambiguous definition of the wallnormal length-scale $h_{\mathrm{EM}}$ is difficult. It represents the vertical extent of the volume within which the EM forces act directly on the flow.

The approach of this question here involves the integration of the EM force over a volume bounded by $L_{\mathrm{E}}, L_{\mathrm{M}}$ and a variable height $y$ (as illustrated in figure 3). Symmetry considerations are such that the resultant of EM forces is

(a)

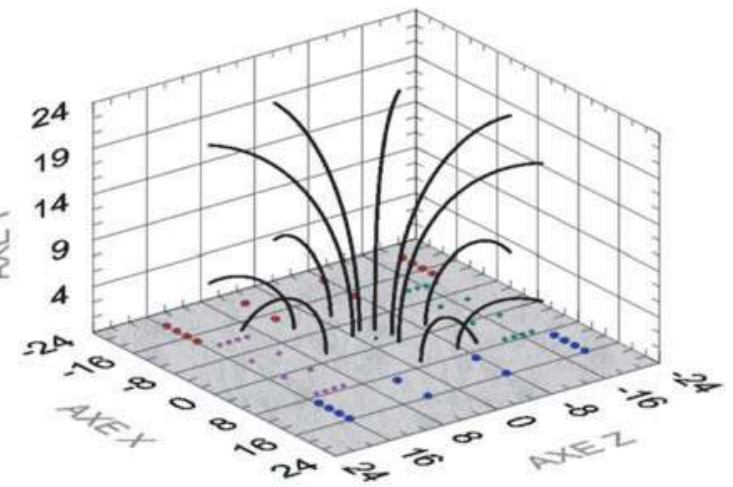

normal to the wall. More precisely, the force distribution presents two planes of symmetry normal to the wall, namely: the one equidistant from electrodes and the one equidistant from the magnets.

Three characteristic parameters of an EM actuator are defined and computed as follows:

(i) Mean EM acceleration $\boldsymbol{g}_{\mathrm{EM}}$ :

$$
\boldsymbol{g}_{\mathrm{EM}}=\frac{1}{\rho v} \int_{v} \boldsymbol{f}_{\mathrm{EM}} d v=\frac{1}{\rho v} \int_{v} \boldsymbol{j} \times \boldsymbol{B} \mathrm{d} v=g_{\mathrm{EM}} \boldsymbol{e}_{\boldsymbol{y}}
$$

$v$ is the volume of integration (figure 3 ); $\boldsymbol{f}_{\mathrm{EM}}$ is the EM body force, $\rho$ the fluid density, $g_{\mathrm{EM}}$ is the mean integral EM acceleration and $\boldsymbol{e}_{y}$ the unit vector perpendicular to the wall.

(ii) $\mathrm{EM}$ velocity, $V_{\mathrm{EM}}$ :

$$
V_{\mathrm{EM}}=\sqrt{2 g_{\mathrm{EM}} y}
$$

This represents the velocity that EM force could produce at a height $y$ from the wall but neglecting viscosity or wall effects.

(iii) Characteristic times:

$$
T_{\mathrm{EM}}=\sqrt{\frac{2 y}{g_{\mathrm{EM}}}}
$$

This $T_{\mathrm{EM}}$ corresponds to the time at which pumping sets in at a height $y$.

Equation (8) can be reorganized as in equation (10). This non-dimensional ratio can be interpreted as the EM equivalent of the Froude number with a value of 1 .

$$
\frac{V_{\mathrm{EM}}^{2}}{2 g_{\mathrm{EM}} y}=1
$$

Due to the sharp drop in the strength of the EM forces, the integrated mean EM acceleration $\left(g_{\mathrm{EM}}\right)$ decreases with $y$. Figure 4 gives $g_{\mathrm{EM}}$ values as a function of $y$.

With this definition, the EM parameters depend on the height of integration, $y$. As a result of the fact that the EM forces decrease rapidly with the distance from the wall (i.e. $y$ ) but never equal zero, the velocity is asymptotically limited as $y$ increases. The EM velocity profile versus $y$ is

(b)

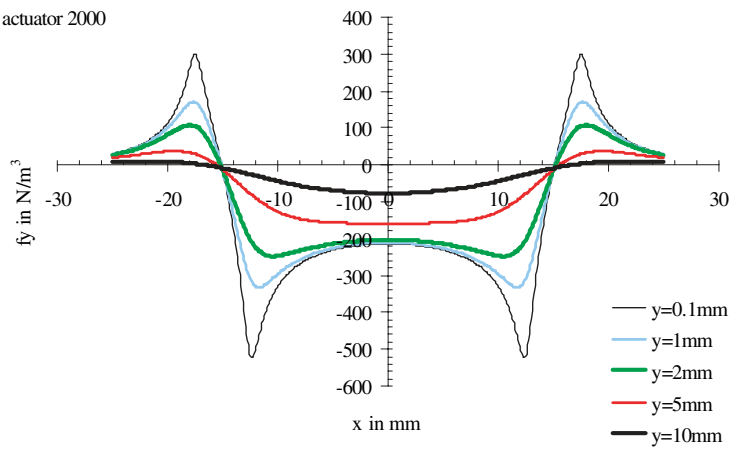

Figure 2. (a) Three-dimensional view of computed EM force lines above an EM actuator set in the $x-z$ plane for $y=0$; $(b)$ variation of the normal component of EM forces, $f_{y}$, as a function of $y$ and $x$ in the plane equidistant to the electrodes. For these computations: $B_{\text {surf }}=1 \mathrm{~T}$ and $I=1 \mathrm{~A}$. 


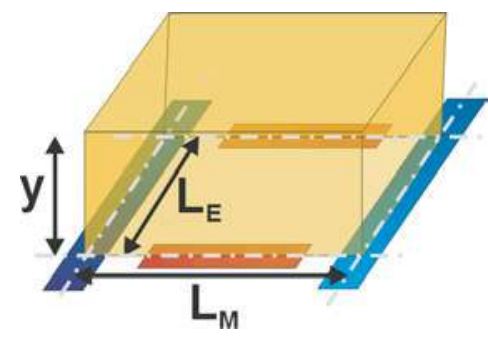

Figure 3. Volume of integration above an EM actuator.

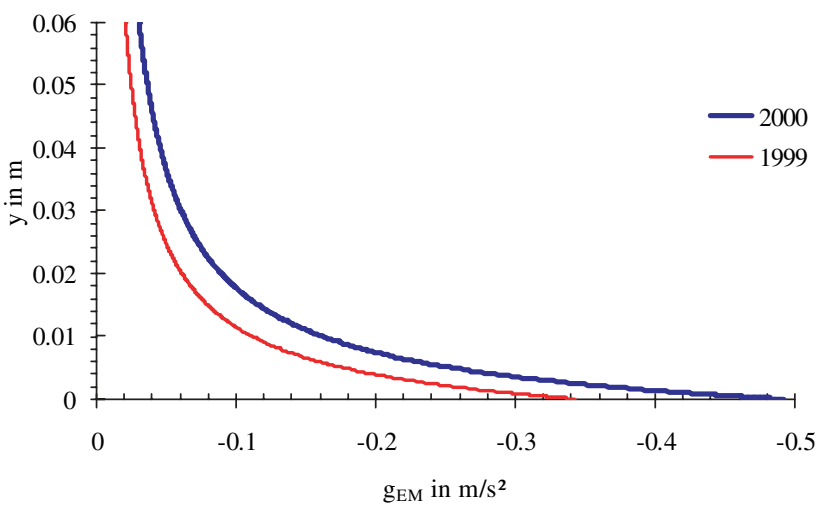

Figure 4. Variation of $g_{\mathrm{EM}}$ as function of $y . B_{\text {surf }}=1 \mathrm{~T}, I=1 \mathrm{~A}$. Two actuators are considered with the same dimensions except the height of the permanent magnet: $8 \mathrm{~mm}$ for the 1999 actuator and $20 \mathrm{~mm}$ for the 2000

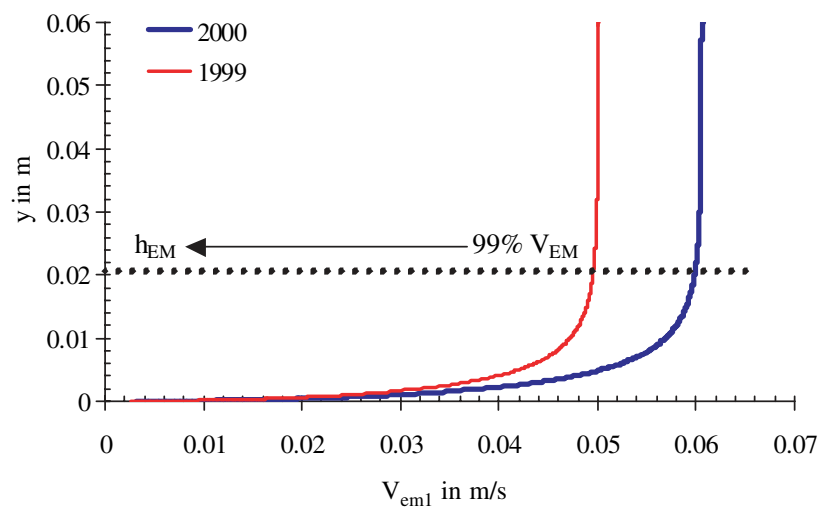

Figure 5. $V_{\mathrm{EM}}$ versus $y$ and illustration of $h_{\mathrm{EM}}$ construction as $y$ for $99 \%$ of maximal $V_{\mathrm{EM}}$. $B_{\text {surf }}=1 \mathrm{~T}, I=1 \mathrm{~A}$.

shown in figure 5. By taking $99 \%$ of this velocity limit, it is possible to define an non-arbitrary integration height and hence a characteristic wall-normal length, $h_{\mathrm{EM}}$, for an EM actuator. This formulation is similar to the one used to define the boundary layer thickness in ordinary fluid mechanics. Here one can give an objective value to height of forcing while the force distribution presents, theoretically, an infinite extent decreasing with increasing distance from the wall. In addition, due to the nature of the EM force, this characteristic length depends only on the EM actuator geometry. The characteristic wall-normal length is therefore independent of current or magnetic intensities, assuming EM forces are still above viscosity damping.

Given this definition of $h_{\mathrm{EM}}$, the EM mean acceleration $g_{\mathrm{EM}}$ and the EM time $T_{\mathrm{EM}}$ can be based on the integration volume with $h_{\mathrm{EM}}$ as height. Since $h_{\mathrm{EM}}$ depends only on the actuator geometry, $g_{\mathrm{EM}}, T_{\mathrm{EM}}$ and $V_{\mathrm{EM}}$ are direct functions of
Table 2. $T_{\mathrm{EM} 1}, g_{\mathrm{EM} 1}, V_{\mathrm{EM} 1}$ and $h_{\mathrm{EM}}$ for actuators 1999 and 2000 and $B_{\text {surf }}=1 \mathrm{~T}$ and $I=1 \mathrm{~A}$

\begin{tabular}{lllll}
\hline & $\begin{array}{l}V_{\mathrm{EM} 1} \\
\left(\mathrm{~m} \mathrm{~s}^{-1}\right)\end{array}$ & $\begin{array}{l}T_{\mathrm{EM} 1} \\
(\mathrm{~s})\end{array}$ & $\begin{array}{l}g_{\mathrm{EM} 1} \\
\left(\mathrm{~m} \mathrm{~s}^{-2}\right)\end{array}$ & $\begin{array}{l}h_{\mathrm{EM}} \\
(\mathrm{m})\end{array}$ \\
\hline Actuator 2000 & 0.0599 & 0.6911 & -0.08669 & 0.0207 \\
Actuator 1999 & 0.0495 & 0.8076 & -0.06133 & 0.02 \\
\hline
\end{tabular}

$I$ (total current) and $\boldsymbol{B}$ (magnetic induction). In table 2, the values with subscript EM1 are reported for a nominal current $I=1 \mathrm{~A}$ and a nominal induction $B=1 \mathrm{~T}$.

The calculated value of $0.8 \mathrm{~s}$ for the characteristic timescale of EM pumping (actuator 1999) is in good agreement with the duration of the transition phase experimentally observed for EM pumping on a flow initially at rest $[4,5]$.

Table 3 gives the laws of dependence of the characteristic EM numbers on the control parameters of an actuator, i.e. $I$ imposed current and $\boldsymbol{B}$ applied magnetic induction.

\subsection{EM Froude similarity}

The pumping effect grows with the intensity of the EM forcing. Using the EM parameters, it is possible to define an EM Froude number appropriate to the flow. This EM Froude number is the ratio of the inertia present in the normal component of the flow (effectively the kinetic energy) to the potential energy in the EM field (effectively the work of EM forces). This expression is

$$
F_{r \mathrm{EM}}=\frac{V^{2}}{2 g_{\mathrm{EM}} h_{\mathrm{EM}}}=\frac{V^{2}}{V_{\mathrm{EM}}^{2}}
$$

A similarity law can be constructed by taking the EM Froude number, $F_{r \mathrm{EM}}$, as a constant in equation (11). When EM forces are above the damping of viscosity, this similarity might be extended to other geometries of actuators considering the pertinent energy and work of forces.

\subsection{Characteristics parameter of various EM regimes}

An EM actuator is designed to act on a flow as it passes through the EM force field. Its actual effect on the flow is expected to depend on the velocity of the fluid as it approaches the domain of action above the actuator.

The characteristic time of the undisturbed flow $T_{\text {flow }}$ can be defined as the transit time of fluid particles over the EM actuator. This is given as

$$
T_{\text {flow }}=\frac{L_{\mathrm{EM}}}{U_{\text {mean }}}
$$

Where $U_{\text {mean }}$ is the undisturbed mean flow velocity and $L_{\mathrm{EM}}$ is the corresponding length of the actuator in the mean flow direction. It is interesting to compare $T_{\text {flow }}$, to the characteristic EM forcing time $T_{\mathrm{EM}}$ (equation (8)):

$$
\mathrm{EM} R=\frac{T_{\text {flow }}}{T_{\mathrm{EM}}}
$$

This non-dimensional time ratio, $\mathrm{EM} R$, presents two asymptotic domains: (i) ${ }_{\mathrm{EM}} R \gg 1$ corresponds to strongly dominant EM forcing, i.e. the imposition of an EM pumping 
Table 3. Estimation of EM characteristic numbers for any current $I$ or induction $B$ deduced from the values computed for $I=1 \mathrm{~A}$, $B_{\text {surf }}=1 \mathrm{~T}$ (i.e. subscript EM1 stands for $I=1 \mathrm{~A}$ and $B=1 \mathrm{~T}$ ) and identical geometries of actuators.

\begin{tabular}{lllll}
\hline$I=1 \mathrm{~A}, B_{\text {surf }}=1 \mathrm{~T}$ & $h_{\mathrm{EM}}$ & $g_{\mathrm{EM} 1}$ & $V_{\mathrm{EM} 1}$ & $T_{\mathrm{EM} 1}$ \\
\hline$I, B_{\text {surf }}$ & $h_{\mathrm{EM}}$ & $g_{\mathrm{EM}}=I B g_{\mathrm{EM} 1}$ & $V_{\mathrm{EM}}=\sqrt{I B V_{\mathrm{EM} 1}}$ & $T_{\mathrm{EM}}=\frac{T_{\mathrm{EM} 1}}{\sqrt{I B}}$ \\
\hline
\end{tabular}

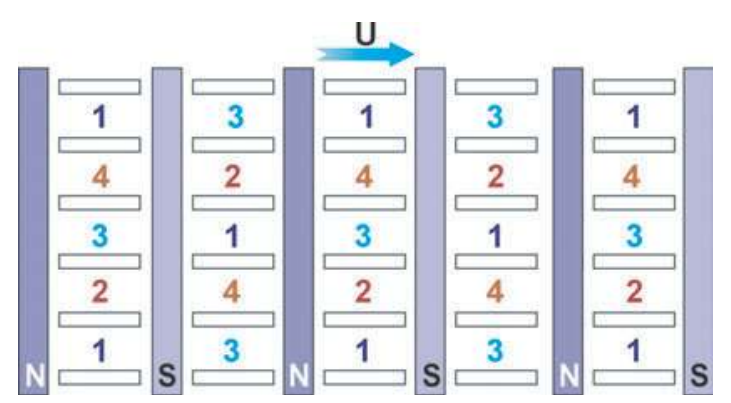

Figure 6. Top view of an EM actuator network with four phases of power supply on a same board.

regime with a single actuator. (ii) ${ }_{\mathrm{EM}} R \ll 1$ corresponds to week EM forcing, i.e. a pumping regime cannot be established using only a single actuator. In this case, the flow will simply be deflected by the generation of a normal velocity component.

\subsection{Multiple activation}

As the flow passes over an EM actuator network (see [1] and figure 6) it undergoes the influence of multiple activations. Each of them has an activation time $T_{\text {act }}$, which cannot be larger than $T_{\text {flow }}$. Between each activations, the flow can recover from the effects of the EM forcing. The following model defines the wall-normal velocity on the $(n+1)$ th actuators as the result of the competition between the relaxation in the flow, represented by $\eta \boldsymbol{V}_{n}$, and the EM forcing, represented by $\alpha \boldsymbol{g}_{\mathrm{EM}} T_{\text {act }}$ with $T_{\text {act }}<T_{\mathrm{EM}} . \quad \eta$ is a relaxation coefficient $(\eta \leqslant 1)$ which is mostly due to viscous dissipation, transport and diffusion. Note that if $T_{\text {flow }}$ is smaller than $T_{\mathrm{EM}}$ (which is the case usually) then $T_{\text {act }}$ is also smaller than $T_{\mathrm{EM}} \cdot \alpha$ is a dissipation coefficient $(\alpha \leqslant 1)$ mostly due to wall effects during the activation. For activation time larger than $T_{\mathrm{EM}}$, via [4] it is possible to give numerical values for $\alpha$ : i.e. $\alpha \sim 0.26$ for the 1999 actuator and $\alpha \sim 0.29$ for the 2000 actuator.

$\boldsymbol{V}_{n+1}=\eta \boldsymbol{V}_{n}+\alpha \boldsymbol{g}_{\mathrm{EM}} T_{\mathrm{act}}$,

$$
\text { with } T_{\text {act }}<T_{\mathrm{EM}} \quad \text { and } \quad T_{\text {act }}<T_{\text {flow }}
$$

In the case of a network (figure 6) with a number of successive actuators, e.g. for an application aimed at drag reduction, a stationary limit velocity $\left(\boldsymbol{V}_{\text {lim }}\right)$ can be defined. This is given by

$$
\boldsymbol{V}_{\mathrm{lim}}=\frac{1}{1-\eta} \alpha \boldsymbol{g}_{\mathrm{EM}} T_{\mathrm{act}}
$$

Clearly, this limit depends on the $\eta$ coefficient. From the energy point of view, the electrical power consumption of an EM actuator network increases with $T_{\text {act }}$. From the physical point of view, the limit of EM flow regime possible corresponds to the time $T_{\mathrm{EM}}$. Consequently, it is not possible to have $\left\|\boldsymbol{V}_{\text {lim }}\right\|>\left\|\boldsymbol{V}_{\mathrm{EM}}\right\|$. Finally, minimum energy consumption implies the following inequality:

$$
T_{\mathrm{act}} \leqslant \frac{(1-\eta) T_{\mathrm{EM}}}{\alpha}
$$

In addition to the flow acceleration, local EM actuators are also a source of vorticity corresponding to the $\operatorname{curl}(\boldsymbol{j} \times \boldsymbol{B})$ (see [4]). In a multi-activation system, like in the network in figure 6, the flow experiences successive activations that are able to modify its vorticity. This alteration (or control) of vorticity is strongly linked to the design of EM actuators as well as to the duty-cycle of the network power supply. Figure 7 shows an illustration $\left(1 \mathrm{~m} \mathrm{~s}^{-1}, R_{\mathrm{ex}}=10^{7}, U_{\infty}=28.6 u_{\tau}\right)$ comparing two classes of actuator sizing: macro- and microactuator. For each case the typical length-scales in wall units including streaks spacing $\left(100^{+}\right)$, streaks size $\left(40^{+}\right)$and streaks vorticity $\left(123 \mathrm{~s}^{-1}\right)$ (see [12]) are compared to the typical intensity of the EM vorticity source. This EM angular acceleration source $\dot{\omega}_{\mathrm{EM}}$ expressed in $\mathrm{s}^{-2}$ is defined as follows:

$$
\dot{\omega}_{\mathrm{EM}}=\frac{1}{\rho v} \int_{v} \operatorname{curl}\left(\boldsymbol{f}_{\mathrm{EM}}\right) \mathrm{d} v
$$

Comparing these two extreme situations, it is clear that the possible modes of action are different. For the macro-actuator, about 1000 wall units (see figure 7(a)), the EM vorticity source $\left( \pm 200 \mathrm{~s}^{-2}\right)$ results in an additional vorticity (typically $\left.\pm 7 \mathrm{~s}^{-1}\right)$, which is of smaller intensity than the pre-existing vorticity.

This means that macro-actuators have to be used in multiple actuation modes. For the micro-actuator, about 100 wall units (figure7 $(b))$ the EM vorticity source $\left( \pm 20000 \mathrm{~s}^{-2}\right.$ ) results in an additional vorticity (typically $\pm 700 \mathrm{~s}^{-1}$ ) which is extremely intense compared to the pre-existing vorticity. This means that a single actuation applied locally is capable of a local control.

\section{Experiments in aquarium and tunnel}

The aim of the experimental investigation is to verify the hypothesis of Froude similarity and to check for the existence of two asymptotic EM regimes. In the experiments, the magnetization of permanent magnets is $1.3 \mathrm{~T}$ and their typical longitudinal length-scale spacing, $L_{\mathrm{EM}}$, is $30 \mathrm{~mm}$.

\subsection{Description of experimental facilities and measurements}

Two measurement methods are used to quantify the flow induced by EM forcing: particle tracking velocimetry (PTV) and particle image velocimetry (PIV). The first series of experiments was carried out on a flow initially at rest in an aquarium large enough to avoid confinement effects. The second series of experiments was performed in a seawater tunnel, with and without external flow. 
(a)

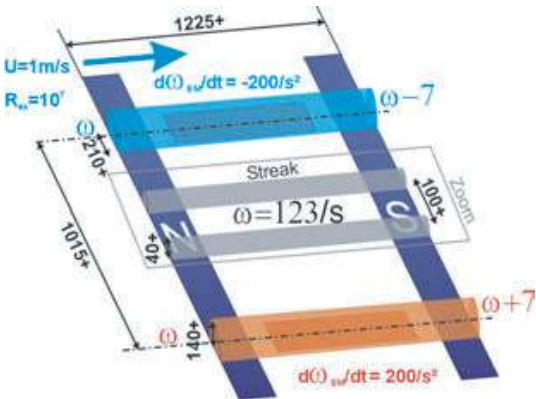

(b)

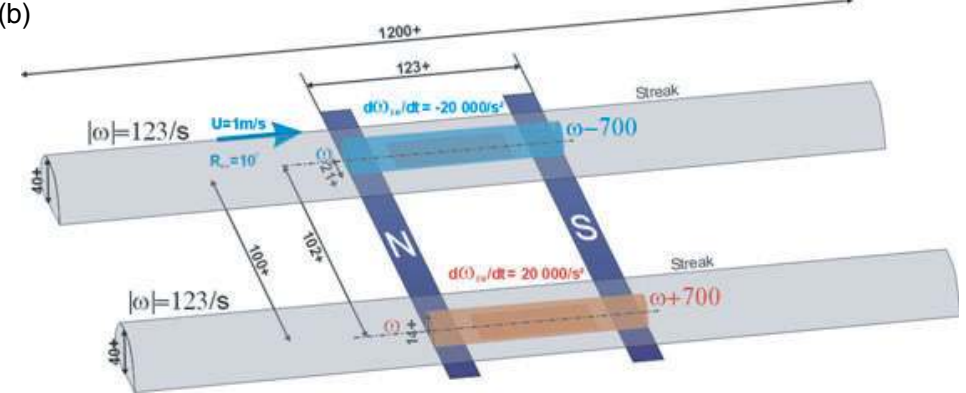

Figure 7. Comparison in wall units of the typical length-scales of an ordinary turbulent boundary layer (streaks spacing and axial vorticity) to the EM vorticity source, $\dot{\omega}_{\mathrm{EM}}$ above the electrodes, for an external velocity of $1 \mathrm{~m} \mathrm{~s}^{-1}, R_{\mathrm{ex}}=107, U_{\infty}=28.6 u_{\tau}$. Two typical actuator length-scales are considered: (a) macro-actuator (about 1000 wall units) with $B=1 \mathrm{~T}$ and $1=1 \mathrm{~A},(b)$ micro-actuator (about 100 wall units) optimized for $T_{\text {flow }}=T_{\mathrm{EM}}$.

(a)

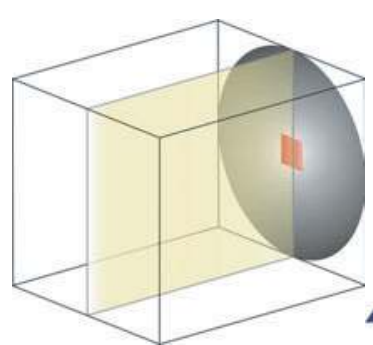

(b)

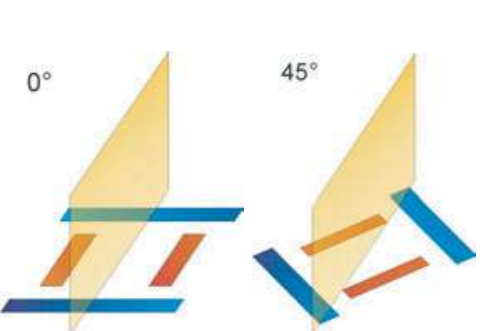

(c)

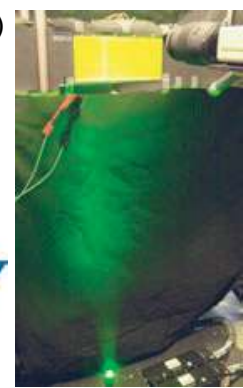

Figure 8. (a) Experimental plane of measurements normal to the wall for the 1999 actuator. (b) Position of the actuator in the aquarium and schematic of the three planes normal to the wall for PTV measurements. (c) Experimental installation for PIV measurements on the tunnel.

\subsubsection{EM activation on a flow initially at rest (PTV} measurements). The aquarium has dimensions of $50 \mathrm{~cm} \times$ $50 \mathrm{~cm} \times 60 \mathrm{~cm}$ and is filled with salt-water $(35 \mathrm{~g} \mathrm{NaCl} / \mathrm{l})$. The EM actuator is situated in the centre of the side vertical wall of the aquarium (figure $8(a)$ ). Figure $8(b)$ shows the relative positions of the laser and light sheets normal to the wall. The dimensions of the aquarium are quite large compared to the size of the EM actuator, i.e. a factor of 20 with $L_{\mathrm{EM}}=30 \mathrm{~mm}$. The EM actuator is activated with a 1 A DC power supply for at least $10 \mathrm{~s}$, a time far greater than the characteristic time of EM forcing $T_{\mathrm{EM}} \sim 0.8 \mathrm{~s}$.

Three planes of measurement (shown in figure $8(b)$ ) are studied in order to specify the flow above the actuator. These wall-normal planes are: (i) $\left(0^{\circ}\right)$ the plane equidistant from the electrodes, (ii) $\left(45^{\circ}\right)$ the plane in the diagonal of the EM actuator and (iii) $\left(90^{\circ}\right)$ the plane equidistant from the magnets.

Figure 9 shows a triple exposure (superposition) image of the flow after a $10 \mathrm{~s}$ activation. This superposition is used for PTV measurements, based on intervals between images of: $0.08 \mathrm{~s}$ and $0.12 \mathrm{~s}$, respectively. Three different electric current intensities of $0.5,0.8$ and $1.1 \mathrm{~A}$ are used. It is then possible to check the Froude similarity concerning currents.

\subsubsection{EM activation on a seawater wall bounded flow (PIV} measurements). These experiments were carried out in a seawater tunnel with a cross sectional area of $100 \mathrm{~mm} \times$ $100 \mathrm{~mm}$ and a test section length of $1.3 \mathrm{~m} \mathrm{[4].} \mathrm{For} \mathrm{all} \mathrm{reported}$ PIV measurements, $y=0$ at the wall and $x=0$ corresponds the centre of the EM actuator. Consequently, the centres of the magnets are situated at $x= \pm 15 \mathrm{~mm}$. Activation is carried out either on originally static fluid and then or on flows

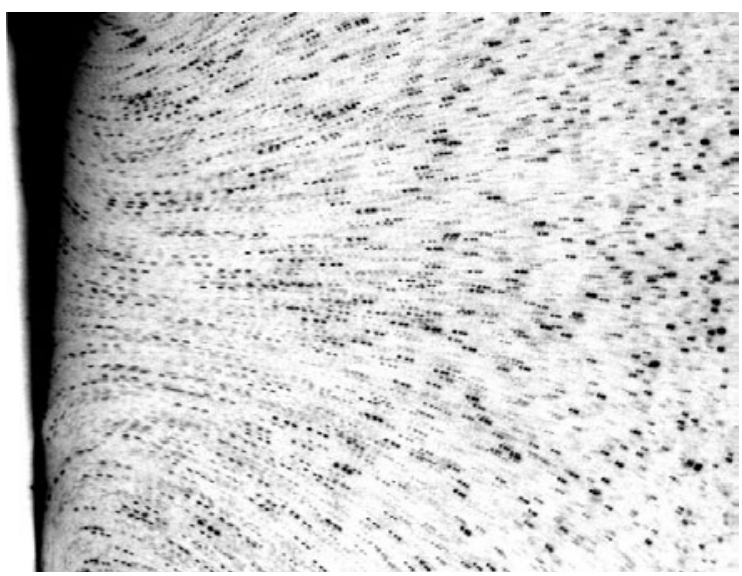

Figure 9. Superposition of three successive images used for PTV measurements.

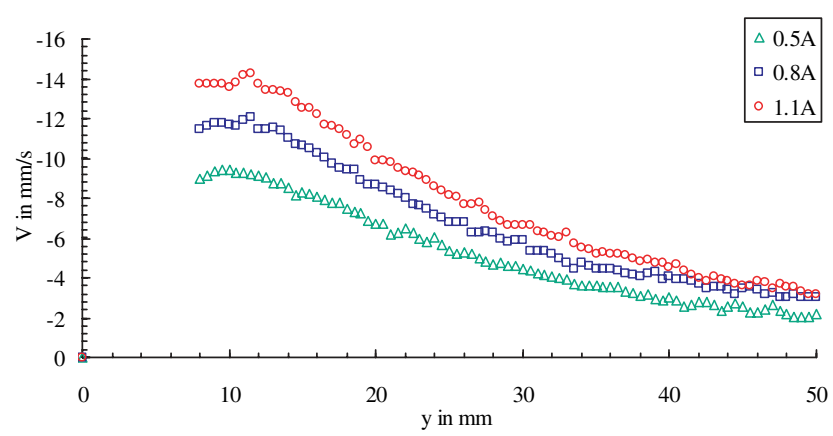

Figure 10. Wall-normal velocity versus $y$ on the axis of the EM actuator for various electric current intensities: $I=0.5,0.8$ and $1.1 \mathrm{~A}$. 
with an imposed external velocity up to a Reynolds number $R_{\text {ex }} \sim 3 \times 10^{5}$.

The PIV measurements are realized just above the EM actuator, which is inserted (wall-flush) in the top wall of the seawater tunnel (see figure 8(c)). Only one wall-normal plane of measurement equidistant from the two electrodes is studied here. This plane, $0^{\circ}$ in figure 8 , is also streamwise to the external flow. A double pulse YAG laser and a digital/numerical camera $\left(1000 \times 1000\right.$ pixels $^{2}$, double frame $)$ are used to take frames. Typically, the delay of acquisition is adjusted for a typical displacement close to $25 \%$ of the size of the cross-correlation window. The cross-correlation windows overlap is $75 \%$ and the ratio of primary to secondary cross correlation peaks is better than 1.2. The various PIV measurements windows are: (i) above the actuator, $40 \mathrm{~mm} \times$ $40 \mathrm{~mm}$ with a resolution of 24.8 pixels $\mathrm{mm}^{-1}$. The size of the correlation windows is 32 pixels, i.e. about $1.3 \mathrm{~mm}$. (ii) Right

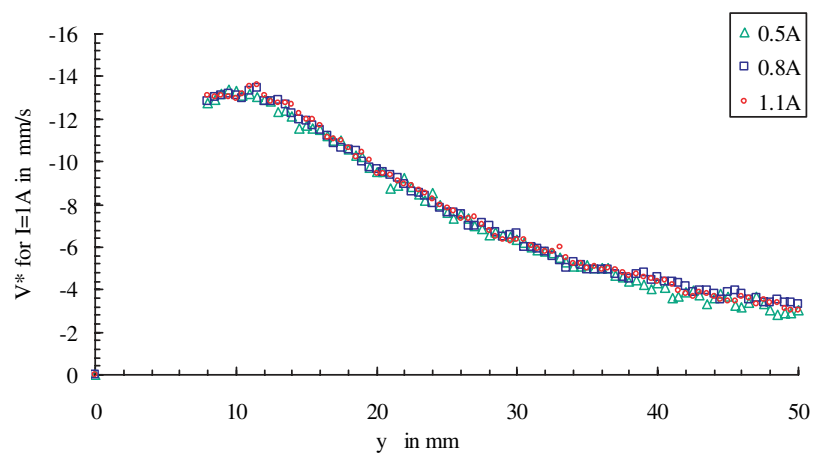

Figure 11. Variation of $V^{*}$ versus $y$ along the axis of the EM actuator. $V^{*}=V \sqrt{I^{*} / I}$ is estimated for $I^{*}=1 \mathrm{~A}$ from experimental points (corresponding to $I=0.5,0.8$ and $1.1 \mathrm{~A}$ ). downstream the actuator, $25 \mathrm{~mm} \times 25 \mathrm{~mm}$, with a resolution of 39.9 pixels $\mathrm{mm}^{-1}$. The typical size of the correlation window downstream the actuator is about $0.8 \mathrm{~mm}$.

\subsection{EM Froude similarity}

4.2.1. Similarity for the 1999 EM actuator and different current intensities. Wall-normal velocity (i.e. velocity component perpendicular to the wall) measurements (PTV) are taken along the wall-normal central axis $y$ of the 1999 EM actuator for three electric current intensities: 0.5, 0.8 and 1.1 A (see figure 10). The curves show the same behaviour. Flow starts from rest far from the wall $(y>60 \mathrm{~mm})$ and is progressively accelerated due to Lorentz force pumping. This acceleration increases with the electric current. The velocity has a plateau type maximum value between 8 and $12 \mathrm{~mm}$. This is attributed to wall effects. Furthermore, $V$ must vanish at the wall, i.e. $y=0$. The intensity of the pumped flow increases with proximity to the wall and with the electric current intensity. Given that the distance over which the Lorentz forces are significant is about $20 \mathrm{~mm}$ far for the actuator, the measurements confirm that the flow resulting from EM activation is able to extend over a larger volume than the forces depending on ${ }_{\mathrm{EM}} R[4,5]$.

Figure 11 presents a simple transposition of the previous curves (figure 10) obtained by dividing the measured velocities by the square root of their electric current intensities, i.e. $V^{*}=V \sqrt{I^{*} / I}$ with $I^{*}=1$ A. All values collapse onto a single curve. This transposition clearly demonstrates the existence of Froude similarity.

The measurements reported in figure 12 are taken at various $y$ positions: $12 \mathrm{~mm}, 18 \mathrm{~mm}$ and $24 \mathrm{~mm}$, and they (a)

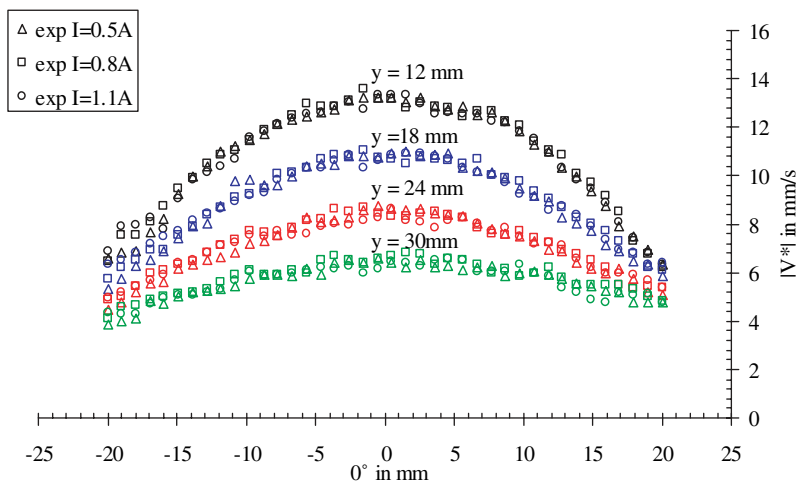

(b)

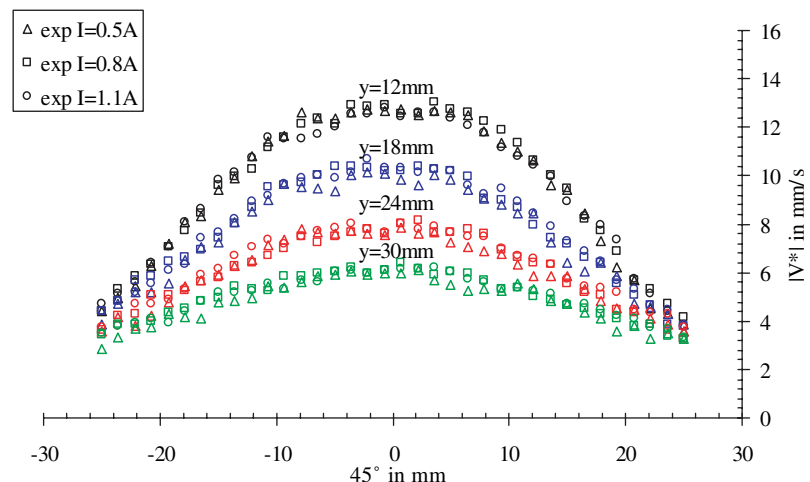

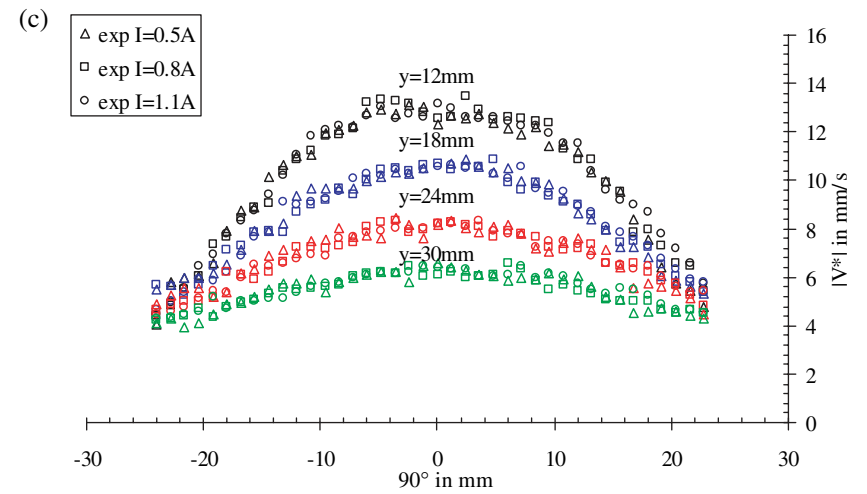

Figure 12. Variation of $V^{*}$ as a function of $y$ for different measurements planes: $(a) \times 0^{\circ},(b) \times 45^{\circ},(c) \times 90^{\circ}$. $V^{*}=V \sqrt{I^{*} / I}$ is estimated for $I^{*}=1 \mathrm{~A}$ from experimental points (corresponding to $I=0.5,0.8$ and $1.1 \mathrm{~A}$ ). 
refer to the three wall-normal planes at $\times 0^{\circ}, \times 45^{\circ}$ and $\times 90^{\circ}$, respectively (cf figure 8 ). Measurements taken at different current intensities are normalized to the corrected current base $I^{*}=1 \mathrm{~A}$ via the $V^{*}=V \sqrt{I^{*} / I}$ similarity law. These wall-normal velocity profiles offer a first quantitative characterization of the wall-normal flow above the actuator. It indicates the intensity of the EM pumping and validates the use of the similarity proposed to various intensity of the forces. By extension, the EM characteristic velocity $\left(V_{\mathrm{EM}}\right)$ scales the potential pumping effect due to Lorentz forces above the actuator. The symmetry of the velocity profile versus $\times 0^{\circ}$, $\times 45^{\circ}$ and $\times 90^{\circ}$ can be noticed. This symmetry is due to the acceleration of the flow and to the axial symmetry of the EM forces (see figure 2).

\subsubsection{Comparison between the 1999 and 2000 actuators.} Different series of experiments with two actuators (1999 and 2000) and under various experimental conditions were carried out both for comparative purposes and for validation of the similarity law. The 1999 actuator was used in an aquarium large enough to allow 'long' activation (up to $10 \mathrm{~s}$ ) before confinement effects became significant. The measurements were taken using PTV. The 2000 actuator was used in the seawater tunnel. The dimensions of the tunnel are necessarily smaller than those of the aquarium. Consequently, the duration of activation was limited to $3 \mathrm{~s}$. For longer activation confinement effects grew strong. Measurements are done by PIV in this case.

Even with these notably different experimental conditions, the normalized measurements (with equation (11)) presented in figure 13 show that the $V^{*}$ profiles effectively collapse onto universal curves for both actuators. This confirms the similarity law proposed for the flow induced by the Lorentz forces. The activation times, respectively, of $3 \mathrm{~s}$ and $10 \mathrm{~s}$ in these experiments, are both larger than $T_{\mathrm{EM}}$. In both cases; behaviour is quite similar confirming that the pumping regime mainly depends on the EM time.

\subsection{EM flow regime}

For the actuator 2000, the flow (of seawater) in the tunnel was set at two different velocity values: 'high velocity'

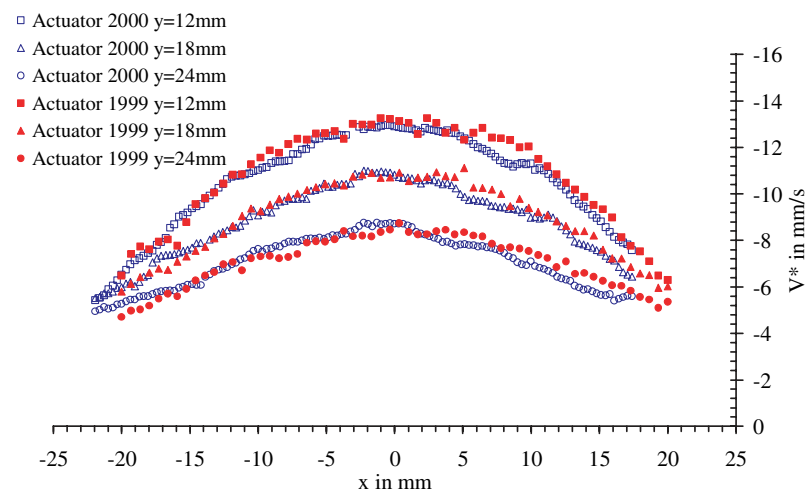

Figure 13. EM Froude similarity between the 2000 and 1999 actuators. $V^{*}$ is estimated from experimental points (corresponding to $I=0.5,0.8$ and $1.1 \mathrm{~A}$ for the 1999 actuator and $I=1.8$ A for the 2000 actuator); for $I^{*}=1 \mathrm{~A}$ and various heights $y: 12,18$ and $24 \mathrm{~mm}$. The measurement plane is equidistant from the electrodes and perpendicular to the wall (see figure $8(b), 0^{\circ}$ ).
$\left(U_{\text {ext }} \sim 100 \mathrm{~mm} \mathrm{~s}^{-1}\right)$ and 'low velocity' $\left(U_{\text {ext }} \sim 10 \mathrm{~mm} \mathrm{~s}^{-1}\right)$. Figure 14 shows the axial velocity profiles taken from PIV measurements. In each case, the velocity profile of the flow without activation is superposed with an EM activated profile.

Clearly, EM activation changes the flow near the wall. The EM forces induce a new flow component in the nearwall flow, which is ascribed to the presence of wall jets. The 'EM' profiles show the presence of wall jets downstream of the actuator. They are characterized by an increase in velocity in the vicinity of the wall and a deficit in axial velocity further out. Figure 15 gives a schematic illustration of this local mechanism.

The very slight drop in the velocity from the maximum value in the unactivated profile for $y>15 \mathrm{~mm}$ (seen in figure 13) is thought to be due to some imperfections in the upstream damping chamber of the seawater loop. This will be modified for future experiments but is not thought to have any influence over the EM flow profiles here.

The wall jets are observed for both 'low velocity' and 'high velocity' flows. In order to give a more accurate meaning to this type of flow classification, with respect to EM pumping, it is interesting to compare the EM time, $T_{\mathrm{EM}}$, with the transit time, $T_{\text {flow }}$ in each case. The ratio of these two timescales is that given in equation (13). When ${ }_{\mathrm{EM}} R \gg 1$ the flow is considered as a case of low velocity and when EM $R \ll 1$ the flow is considered as a case of high velocity. Classification of the flows according to the $\mathrm{EM} R$ values is
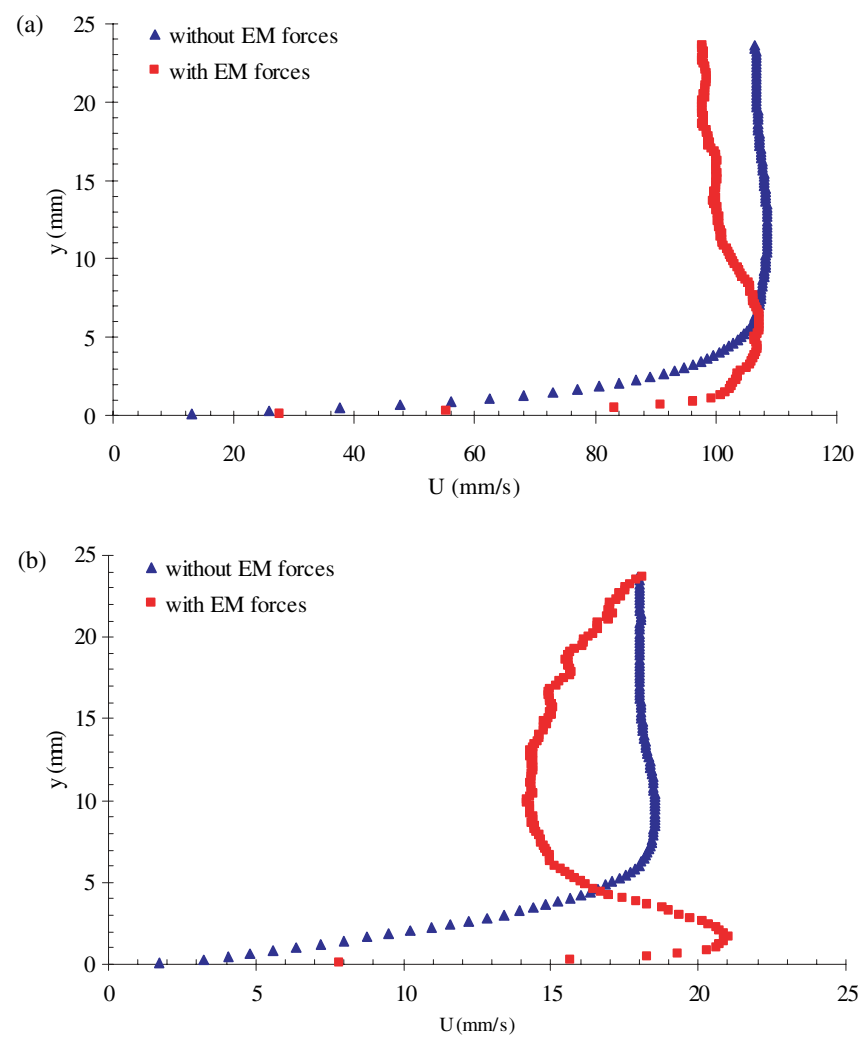

Figure 14. Axial velocity profiles downstream of the EM actuator $(x \sim 37 \mathrm{~mm}$ from the axis). $\mathbf{\square}$ : with EM forces and $\mathbf{\Delta}$ : without EM forces. (a) Mean velocity (without EM forces) is about $101.5 \mathrm{~mm} \mathrm{~s}^{-1}$. Activation current is $1.78 \mathrm{~A}$; (b) mean velocity (without EM forces) is about $16.6 \mathrm{~mm} \mathrm{~s}^{-1}$, activation current is $\sim 1.73 \mathrm{~A}$. 
used on the series of measurements presented in figures 16 and 17.

EM $R>1$ : (this case corresponds to an external velocity of $16.6 \mathrm{~mm} \mathrm{~s}^{-1}$ ) (see figure 17(a)). The transit time of the fluid particles is about $T_{\text {flow }}=1.8 \mathrm{~s}$ and the EM forcing time is about $T_{\mathrm{EM}}=0.65 \mathrm{~s}(I=1.73 \mathrm{~A})$. The flow is clearly accelerated near the wall. The velocity change $\left(u-u_{\text {ref }}\right)$, close to the

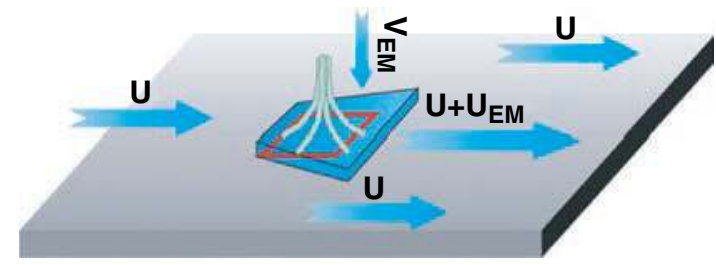

Figure 15. Illustration of wall jets downstream of the EM actuator.

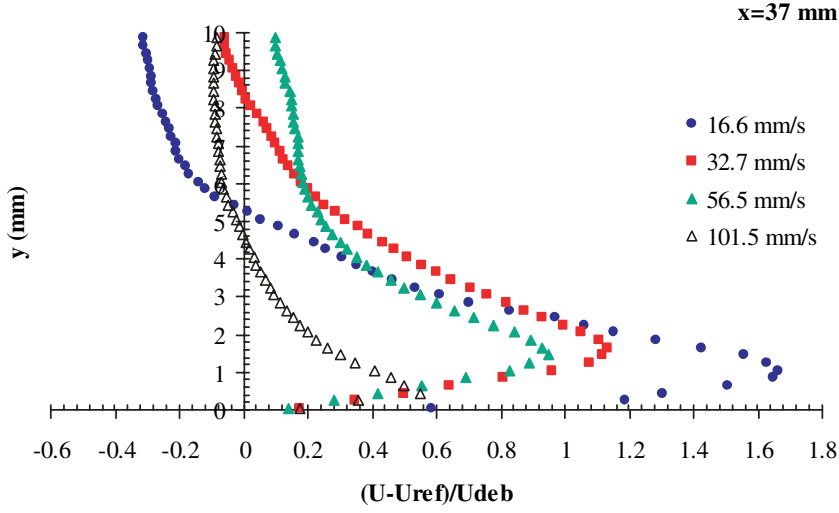

Figure 16. Non-dimensional axial velocity profiles $\left(U-U_{\text {ref }}\right) / U_{\text {deb }}$ normal to the wall downstream of the EM actuator $(x \sim 37 \mathrm{~mm}$ from the axis) for four mean velocities: $16.6,32.7,56.5$,

$101.5 \mathrm{~mm} \mathrm{~s}^{-1}$. $U$ is the velocity with EM forces, $U_{\text {ref }}$, the velocity without EM forces and $U_{\mathrm{deb}}$, the mean velocity is defined as flow rate/cross-sectional area. wall, is found to be significantly larger than the mean flow velocity defined by the flow rate/cross-sectional area of the tunnel (see figure 16). Its maximum is at $y=1.3 \mathrm{~mm}$ from the wall. Clearly, in this case the wall jet dominates the flow near the wall.

$\mathrm{EM} R<1$ : (this case corresponds to the velocity of $101.5 \mathrm{~mm} \mathrm{~s}^{-1}$ ) (see figure 17(b)). The transit time of the fluid particles is about $T_{\text {flow }}=0.3 \mathrm{~s}$ and the EM forcing time is about $T_{\mathrm{EM}}=0.64 \mathrm{~s}(I=1.78 \mathrm{~A})$. The competition between the axial mean flow and the EM pumping (normal to the wall) is such that wall jets are less well defined and the flow is mainly deflected to the wall.

Finally, the time ratio $\mathrm{EM}^{R}$ can be generalized to asymptotic domains: $\mathrm{EM} R \gg 1$ corresponds to 'pumping mechanism' and ${ }_{\mathrm{EM}} R \ll 1$ corresponds to 'deflecting mechanism' of EM force effects.

\section{Conclusion}

The ability of EM actuators to generate novel flows inducing strong changes in the near wall flow has been demonstrated. The forces developed above the EM actuator considered in this paper are mainly normal to the wall above the central axis of the actuator; in addition, the curl of these forces acts as a vorticity source for the flow. Depending on the intensity of the EM forces relative to the initial flow conditions, the flow can be deflected or effectively pumped to the wall.

The inertial model proposed here is based on an integrated EM acceleration. This model leads to the identification of EM characteristic numbers such as EM velocity, EM forcing time and EM normal length. It is remarkable that these numbers are independent of the flow length-scales depending only on the actuators length-scales. A group of non-dimensional parameters is constructed from these characteristic numbers:

(i) The EM Froude number is identified as the most important regarding similarity between various experiments. This is

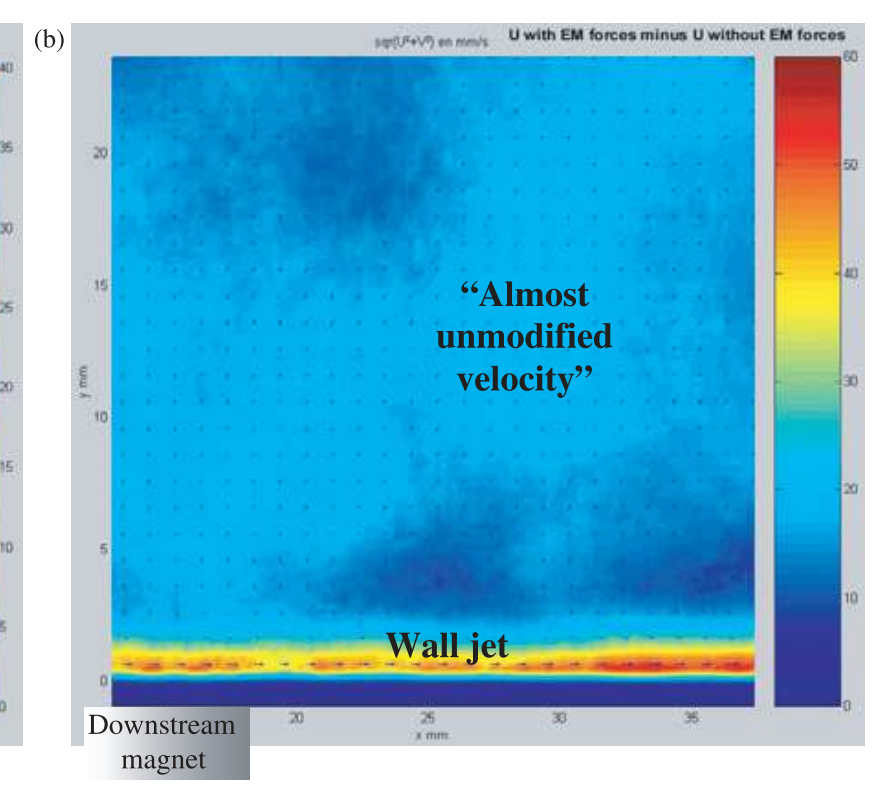

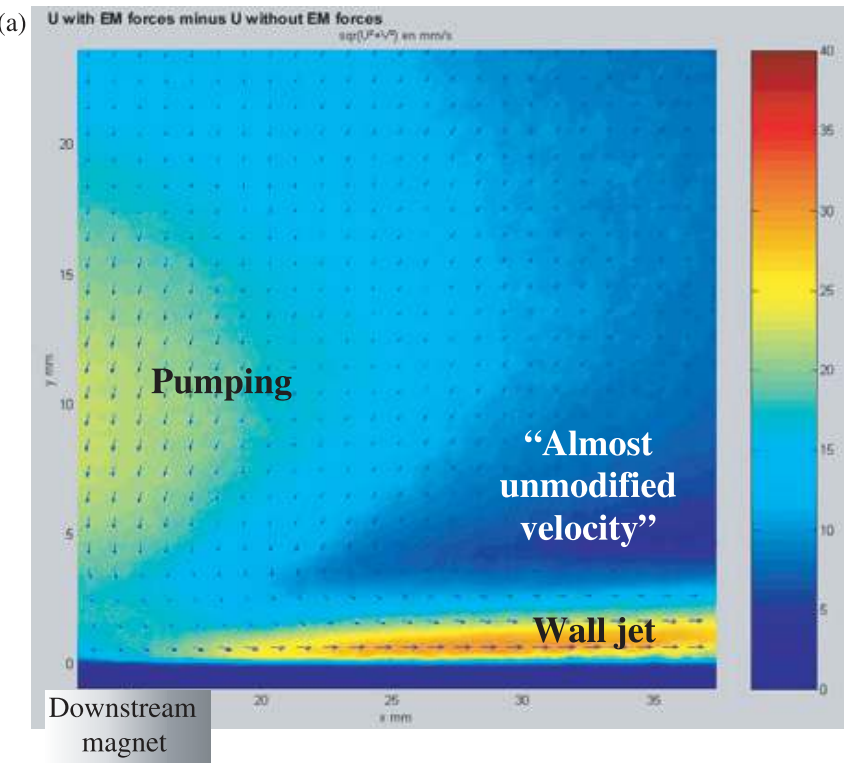

Figure 17. PIV plot of the velocity difference $\left\|u-u_{\text {ref }}\right\|$ in $\mathrm{mm} \mathrm{s}^{-1}$, for two different mean velocities $U_{\text {mean }}$. (a) Low velocity: $U_{\text {mean }}=16.6 \mathrm{~mm} \mathrm{~s}^{-1}$ and current: $I \sim 1.73 \mathrm{~A} ;(b)$ high velocity: $U_{\text {mean }}=101.5 \mathrm{~mm} \mathrm{~s}^{-1}$ and current: $I \sim 1.78 \mathrm{~A}$. 
well confirmed by measurements where this EM Froude number appears to control the pumping of the flow by Lorentz forces.

(ii) The EM time ratio allows to distinguish between EM forcing regimes. When the EM time is smaller than the transit time (i.e. EM ratio larger than 1), the flow is strongly pumped to the wall and is entirely dominated by the EM forces. The relative energetic price of this kind of activation is quite high. When the EM time is larger than the transit time (i.e. EM ratio smaller than 1), the flow is deflected to the wall. In both cases, a resulting novel wall flow is observed (i.e. wall jets), which is capable of reorganizing the near-wall flow and consequently modifying the production of turbulence.

The analysis presented here is validated by measurements. It has to be considered as a guideline for any attempt to optimize the use of single or multiple EM actuators.

\section{Acknowledgments}

This work has been carried out with the support from DGA/BEC and DGA/DSP (departments of the French Ministry of Defense). Many thanks for extensive discussions and contribution to Dr F M J Mc Cluskey, Dr S Tardu, P Carrechio and M Kusulga of LEGI; 'service repro-visu' of the ENSHMG and his director Mr F Bonnel; Dr O Cugat and Dr J Delamare of LEG-ENSIEG.

\section{References}

[1] Nosenchuck D M and Brown G L 1993 The direct control of wall shear stress in a turbulent boundary layer Proc. Int. Conf. on Near Wall Turbulent Flows (Amsterdam: Elsevier) pp 689-98
[2] Henoch C and Stace J 1995 Experimental investigation of a salt water turbulent boundary layer modified by an applied streamwise magnetohydrodynamic body force Phys. Fluids 7 1371-83

[3] Weier T, Fey, Gerbeth G, Mutschke G and Avilov 2000 Boundary layer control by means of electromagnetic forces ERCOFTAC Bulletin No 44, mars 2000, pp 37-41

[4] Rossi L 2001 Contrôle électromagnétique d'écoulement en eau de mer $P h D$ Theses Université Joseph Fourier Grenoble, October 2001

[5] Rossi L and Thibault J-P ElectroMagnetic forcing in turbulence and flow control: analytical definitions of EM parameters and hydrodynamic characterisations of the flow Phys. Fluids submitted

[6] Rossi L and Thibault J-P 2002 Investigation of wall normal electromagnetic actuator for seawater flow control J. Turbulence 3005

[7] Robinson 1991 Coherent motions in the turbulent boundary layer Ann. Rev. Fluid. Mech. 23 601-39

[8] Adrian R J, Tomkins C D and Meinhart C D 2000 Vortex organization in the outer region of the boundary layer $J F M$ 422 1-54

[9] Meng J 1998 Engineering insight of near-wall microturbulence for drag reduction and derivation of a design map for seawater electromagnetic turbulence control Proc. Int. Symp. on Seawater Drag Reduction (Newport R.I.) pp 389-93

[10] Zhou J, Adrian R J, Balachandar S and Kendall T M 1999 Mechanisms for generating coherent packets of hairpin vortices in channel flow JFM 387 353-96

[11] Akoun G and Yonnet J-P 1984 3D analytical calculation of the forces exerted between two cuboidal magnets IEEE Trans. Magn. 20

[12] Meng J C S 1996 Wall layer microturbulence phenomenological model and a semi markov probability predictive model for active control of turbulent boundary layers NUWC Division Newport Technical Digest 1996

[13] Boissonneau P and Thibault J P 1999 Experimental analysis of couplings between electrolysis and hydrodynamics in the context of MHD in seawater J. Phys. D: Appl. Phys. 32 2387-98 